\title{
An Empirical Study on Fake News Detection System using Deep and Machine Learning Ensemble Techniques
}

\author{
T V Divya ${ }^{1}$ \\ Research Scholar, Department of Computer Science and \\ Engineering, KoneruLakshmaiah Education Foundation \\ Aziz Nagar, Hyderabad, Telangana
}

\begin{abstract}
With the revolution that happened in electronic gadgets in the past few years, information sharing has evolved into a new era that can spread the news globally in a fraction of minutes, either through yellow media or through satellite communication without any proper authentication. At the same time, all of us are aware that with the increase of different social media platforms, many organizations try to grab people's attention by creating fake news about celebrities, politicians (or) politics, branded products, and others. There are three ways to generate fake news: tampering with an image using advanced morphing tools; this is generally a popular technique while posting phony information about the celebrities (or) cybercrimes related to women. The second one deals with the reposting of the old happenings with new fake content injected into it. For example, in generally few social media platforms either to increase their TRP ratings or to expand their subscribers, they create old news that happened somewhere years ago as latest one with new fake content like by changing the date, time, locations, and other important information and tries to make them viral across the globe. The third one deals with the image/video real happened at an event or place, but media try to change the content with a false claim instead of the original one that occurred. A few decades back, researchers started working on fake news detection topics with the help of textual data. In the recent era, few researchers worked on images and text data using traditional and ensemble deep and machine learning algorithms, but they either suffer from overfitting problems due to insufficient data or unable to extract the complex semantic relations between documents. The proposed system designs a transfer learning environment where Neural Style Transfer Learning takes care of the size and quality of the datasets. It also enhances the auto-encoders by customizing the hidden layers to handle complex problems in the real world.
\end{abstract}

Keywords-Transfer learning; GANS; glove algorithms; word2vec; ensemble techniques; auto encoders; pre-trained models; word embeddings; BERT models

\section{INTRODUCTION}

A few decades back, researchers started working on fake news detection topics with the help of textual data. In the recent era, few researchers worked on images and text data using traditional and ensemble deep and machine learning algorithms, but they either suffer from overfitting problems due to insufficient data or unable to extract the complex semantic relations between documents. The proposed system

\author{
Dr Barnali Gupta Banik ${ }^{2}$ \\ Associate Professor, Department of Computer Science and \\ Engineering, KoneruLakshmaiah Education Foundation \\ Aziz Nagar, Hyderabad, Telangana
}

designs a transfer learning environment where Neural Style Transfer Learning takes care of the size and quality of the datasets. It also enhances the auto-encoders by customizing the hidden layers to handle complex problems in the real world. The identification of fake news or phony news does treat as a sort of "Spam Detection," for which the reliability or accuracy of the model generally depends on the processing of the textual and image data. In this section, article will discuss a few popular textual and image pre-processing techniques. Initially, for the multi-media content, system have to work with images first. The dataset consists of three types of images, namely, a) Fake Image (tampered or morphed), b) Pristine Image (Resizing operation does perform to maintain standard size for all images), c) Image Splicing (it adds new content to the image either in image or text). The samples of fake news about celebrities that are have posted on Facebook does represent in Fig. 1. One of the fake news here is posted about celebrity with name "Tina Turner" stating that she is one of the top most among the haters list released by reputed organization, which is later found to be false. Like this many fake stories are released by few media and social networks to get the attention of viewers. The popular example for these type of examples is you tube people post false thumbnails on their videos irrespective of the content that might be displayed in the videos.

Any algorithm for working with these type of images, any model first need to separate the text and image as shown in Fig. 2.

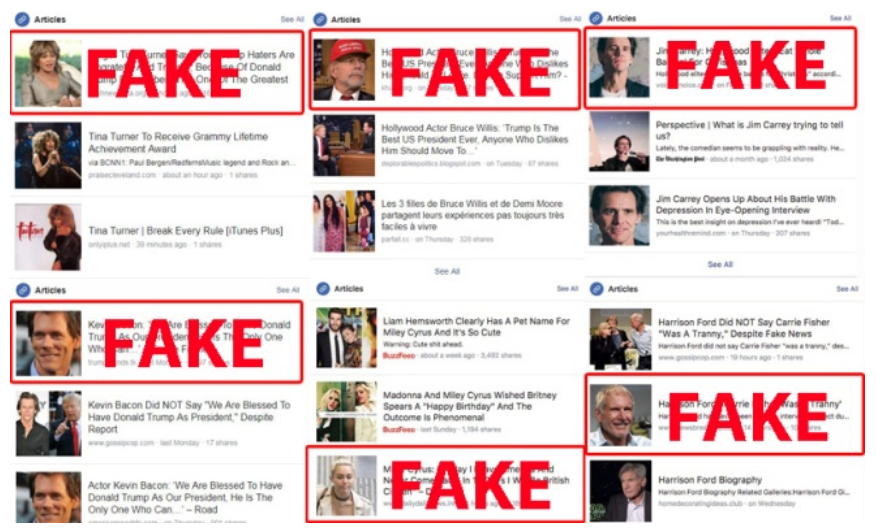

Fig. 1. Fake News Published on Facebook. 


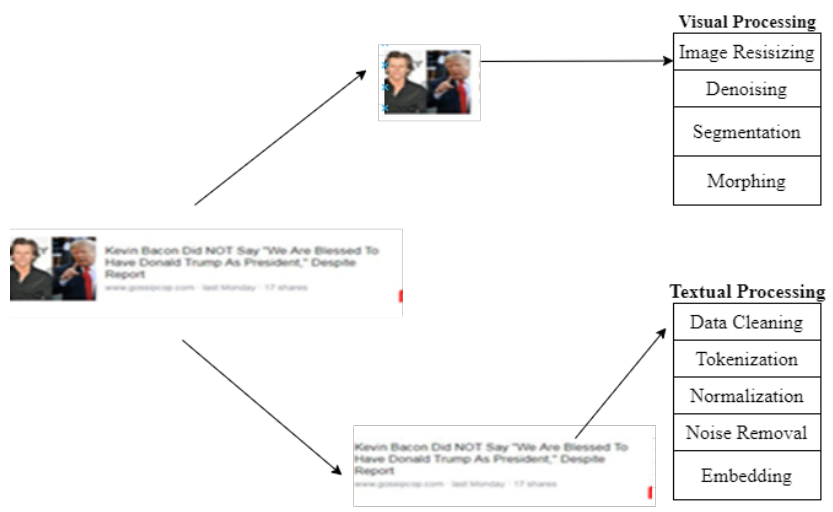

Fig. 2. Working with Multi-Media Content.

\section{A. Image Pre-processing Techniques}

This section will discuss mainly four types of processing techniques, as shown in Fig. 2. The image resizing does perform to standardize all the images to equal width and height because different articles will have different dimensions, then it becomes difficult for the neural network to give training based on the dimensions. The Denoise removal of noise steps helps the system improve the image's quality, majorly for the images captured with a low-quality lens or blur images posted due to the motion that occurred while capturing the image. Noises can exist in multiple forms, as shown in Fig. 3.

The segmentation process [22] helps find the interesting regions in the image to subtract the unwanted or uninterested parts from the original image. This process reduces the complexity as well as time to work with the image. The major pre-processing in the phony news detection system is finding the linkage or sequence in between the articles with the same headlines or continuing to a news article published a long back. These scenarios are taken care of by defining the transformation and morphing points or lines across the different images available in the dataset. All these methods mentioned are traditional, so to achieve an excellent accurate system, the researchers can replace these mechanisms with deep learning techniques like CNN [23], LSTM, and others, which are described in Table I.

Apart from these possible techniques, the best way to determine the quality of model is usage of GAN's to generate the duplicate images of different orientations by defining two types of layers one for generator and second layer for discriminator. The purpose of the generator is to create more number of relevant images and discriminator tries to identify the fake images.

\section{B. Related Knowledge}

In the implementation of GAN, there exists two components, namely, generator and discriminator. The major focus of the generator is to make the discriminator to believe the information generated about the fake images it has created. Since, the proposed model involves both text and images; the best case of GAN to implement is neural style GAN [21] because style GANS majorly focus on the tampered or morphed images rather than the real objects that exists in the image. The shape, color and edges are the crucial elements in the image processing. The extraction of these features should not impact the other layers of the network, so it's better to take the help from encoding mechanisms.

\section{Text Pre-processing Techniques}

In this section, the article discusses the basic techniques of NLP to deal with textual data extracted from the image. Data cleaning deals with removing stop and abuse words from the content, handling special characters, emojis, performing either stemming or lemmatization so that important base words are maintained, and others eliminate them from the content. The next step is to count the occurrences or frequencies in various new articles starting from character to sentence because the higher the frequency higher the priority to maintain the element in the content. The crucial text processing step is normalization because the semantic relation between the words (or) sentences does establish. The popular normalization technique is "POS Tagging," which labels each word in the sentence with parts of speech so that all the words might not be reduced, and sometimes over lemmatization might change the meaning of the entire sentence. The word embedding tries to find similar words with different representations and merge them to have a unique representation in the entire article so that the reader finds fewer complications with the interchange of similar words. The overall tentative proposed system does represent in Fig. 4, which can solve the major difficulties identified in the previous systems.

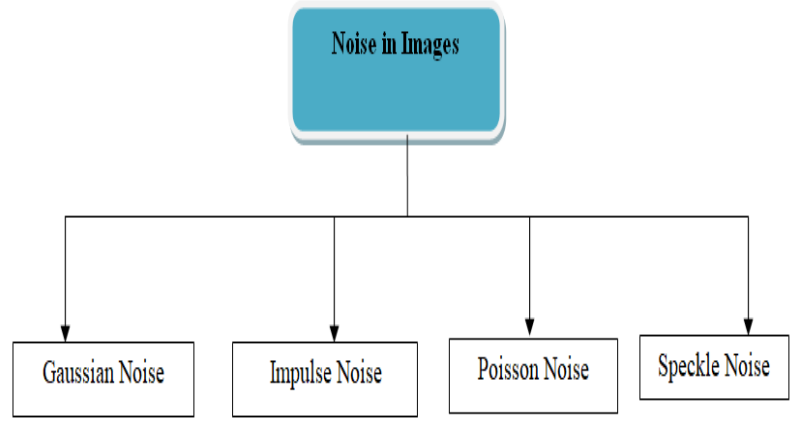

Fig. 3. Types of Noises in Image Processing.

TABLE I. DESCRIPTION OF NEURAL NETWORK TECHNIQUES

\begin{tabular}{|l|l|l|}
\hline S.NO & Name & Description \\
\hline 1 & CNN & $\begin{array}{l}\text { In general images are represented in the form of 2D } \\
\text { matrix, to explore all possible features; the CNN } \\
\text { represents them as a 3D matrix. The extraction of } \\
\text { features are performed with the help of hidden } \\
\text { layers }\end{array}$ \\
\hline 2 & LSTM & $\begin{array}{l}\text { It tries to identify the features from the previous } \\
\text { predictions which are arranged in an sequential } \\
\text { manner taking the help of circuit gates }\end{array}$ \\
\hline 3 & MLP & $\begin{array}{l}\text { It is a fully connected layer and data units are } \\
\text { transferred through all the layers for processing. } \\
\text { These type of networks are applied for applications } \\
\text { where speech act as input and to work with complex } \\
\text { classification systems }\end{array}$ \\
\hline 4 & RBFNN & $\begin{array}{l}\text { In this model, the input vectors are compared to find } \\
\text { the similar data points and every vector is compared } \\
\text { against all the possible classes of the neural } \\
\text { networks. }\end{array}$ \\
\hline
\end{tabular}




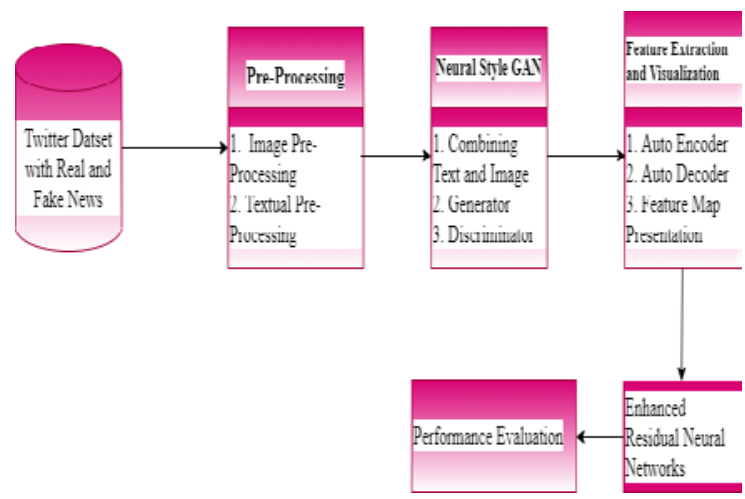

Fig. 4. Brief Overview of the Fake News Detection System.

In the below sections, the paper is organized as follows, the literature survey provides a glance of all the previous existing mechanisms and results and discussion section discusses their advantages and disadvantages to find the limitations that exists in the previous works along the datasets they have used. The observations section identifies the accuracies of all the models and describes about the best model it identified.

\section{LITERATURE SURVEY}

In [1], Xinyi Zhou et al. explored a multi-modal analysis to detect fake news using both text and images. First, it extracts the information from the image and runs the similarity matching algorithm with different types of contents. The textual features extract using the text-CNN, in which a fully connected layer does attach to the normal CNN, and the important contents did extract after passing through each layer. For processing the images, a pre-trained model known as "image2sentence" is implemented. An independent model is developed by performing the cross-entropy modal. Every image does label with multiple classes, and the probability distribution function access the correct prediction label by using the concept of majority voting schema.

In [2], Priyanshi Shah et al. designed a framework by analyzing the tweets, which are made available in public datasets. The textual extraction performs sentiment analysis, i.e., by identifying the polarity value for all the tweets. In visual extraction, segmentation is applied by combining wavelets with K-Means and extracting nine important images. The features extracted from text and visual are represented as a matrix and passed as input to the optimal algorithm proposed by the author. Then it identifies the relevant contents and is passed to the SVM classifier with RGBF kernel function to classify the image.

In [3], Anastasia Giachanou et al. have studied multimodel evaluation using two datasets and by combining the neural networks with semantic search. The tags on images were observed and did pass as input for the local binary patterns algorithm, which finds the similarity between the text and images. The model can identify the different images with the same content. In [4], Deepak Mangal et al. proposes text cues to extract features from images by implementing the LSTM technique in neural networks. It removes the information from tag line and compares it with various news articles by using the cosine similarity mechanism. The visual features do train using VGG, and text features do train using word2vect corpus. The LSTM uses three gates to generate the classification by using useful information. The non-linearity function helps the layer identify any short sequences that occur but has a great role to play in predicting.

In [5], Tong Zhang presented a novel approach known as "BERT" implementation in neural networks for identifying the essential domain features. In this model, a component called "BDANN" identifies irrelevant features and integrates with the remaining modules to focus on the event-specific tasks. The model works in both forward and backward directions, and it works on the domain classifier by making them into sub-groups with defining specific patterns for each group to identify in the future quickly. This model has achieved an accuracy of $98.1 \%$ and presented well even in the case of true label prediction.

In [6], SomyaRanjanSahoo et al. experimented with an automatic system that can detect multiple features to identify the fake news posted on social networks. This model first analyzes the profiles of the users through a web crawler attached to the browser to get the features associated with the user. These extracted features apply machine learning like KNN, SVM, Logistic Regression, and deep learning techniques like LSTM to classify real and fake news. The model has divided features into two significant categories: contents related to profile and contents newly created, and tries to generate a mapping technique between the previous posts and new ones.

In [7], Aman Agarwal et al. explored a new approach in neural networks by blending different deep learning algorithms. The model tries to establish a relationship in terms of language by constructing a word vector using a novel algorithm known as "GLOVE" to process the textual data. The beauty of word embedding lies in its representation of sentimental meaning and their corresponding relation in geometric shapes, which helps visualize the vector space. The reason behind this shape utilization is, it marks how far two different words did match one against the other. Later, these different vector spaces are organized into a bag of words, using Keras as a pre-trained model to construct a sequential model. The major work in this system focuses on turning the parameters associated with convolution and recurrent neural networks. Using the CNN model and embedded trained matrix, N-gram higher-order representation does construct to find the co-relations between the different sequences to construct a feature vector map. With this matrix, there is a possibility of obtaining non-linear transformations in the model, which the LSTM helps classify the temporal and essential features among the text data available. This model also helps in handling the unlucky data that might be present in the model by any chance by defining the threshold values, by adding the generalized layers like dropout, normalizations, and others.

In [8], Juan Cao et al. discussed detecting fake news in different multi-media channels. The model has designed deep learning techniques incorporated with image processing compression techniques to identify the manipulations or 
tampered text within the images. The major role of CNN is to extract the semantic and content features associated with the images, and later it performs statistical measures to perform feature engineering. In this system of adversarial image reconstruction, the first part of the algorithm deals with metadata generation from the top k-features extracted from the reference dataset, and the second part of the algorithm act as a consistency verifier to generate the evidence that is against the tampered image because it is essential to know the relationship between the image and text to make some inferences and decisions.

In [9], Yin-Fu Huang et al. modeled an Adaptive Harmony based genetic algorithm using ensemble techniques of deep learning. The highlight associated with this model is, it has successfully processed data based on their dimensions. For example, suppose the tokenization of words has to perform, then the embedded LSTM takes care by extracting the semantics in the form of 300 dimensions. Similarly, the depth of the sentence and parsing of grammar is taken care of by depth LSTM. From these different LSTM's, an optimized LSTM with minimum weights is constructed. The classification of news is taken care of by heuristic genetic algorithm, which consists of first random selection, whose performance computed using the objective function, which defines as the minimum weight of the output node then in the second step, the value of the node updates if and only if the memory consideration accepted. In every iteration, it adjusts the pitch such that every time it selects the elements that are less than the threshold value and simultaneously reduces the loss function by wrapping the MSE error function with a harmony algorithm.

In [10], AnshikaChoudhary et al. designed a framework based on language to detect fake news. This system uniquely concentrates on syntax related to the sentence instead of semantics by computing the density of each word. During this process, it takes three statistical measures into account; one is count, which computes the occurrence of each word, second one deal with sentences to extract the sentiment associated with it in the form of polarity, which represents the negative and positive words and subjectivity, which assigns the score as either 0 or 1 . The last one takes care of the properties associated with grammar by computing the readability of the sentence based on the pattern of writing. In deep learning algorithms, all the features are standardized and passed as input to the network with two hidden layers, which designs a variant of LSTM by taking the input gate's input because it determines the number of updated values do add to the four neurons. The layer might feel some of the features are unimportant in the next phase, so the forget gate can control this. Finally, using the activation function tanh and the dot product of weighted sums, the memory gate computes the final value at the output node.

In [11], JiangfengZeng computed deep semantic correlations between the textual data and multi-media images by using pre-trained models like VGG to deal with variations in hierarchical representation systems. The model knows the complexities involved in training with the machine learning model, so it has opted for the computation of Eigenvectors to train the model, which in turn helps in the image enhancement process. The system takes news articles, i.e., text documents and the image, associated with annotated labels as input. The system uses the concept of word embedding for processing the textual data, for converting the words which are semantically closer as a group of vectors, and VGG-19 to train the features related to the images in the transfer learning environment because, in the end, they produce the output as an encoded image. The bi-directional LSTM integrated with soft attention encodes the word to extract the complete sentence in terms of grammar and other notations and represents them in a hierarchal format known as "DOM"; these can traverse in both directions. At last, the visual content connects with two dense, fully connected layers to perform decoding of both textual and visual features.

In [12], Rohit Kumar Kaliyar et al. designed a tensor decomposed matrix to work with the BuzzFeed dataset by integrating the machine learning algorithm "eXtreme Gradient Boost" algorithm with a proposed algorithm known as "DeepFake." This system involves only detecting fake in terms of social context, the construction of which needs two major inputs is contents related to the article, and the second is a matrix that defines the relation of users with different communities. The contents convert into an N-grams count vector, representing the occurrence of a particular word in different news articles. The relationship matrix converts into couple matrix factorization and mode- 1 participation by constructing echo chambers, where people share their opinions about it a particular post. These matrices help compute the interests of different communities based on their comments and relationships in the network. The DeepFake architecture has designed a 4-layered network with correct regularizations.

In [13], Muhammad Umer et al. experimented with stance detection using multi-classification techniques with the help of neural networks. The headlines associated with the news article are converted into a 100-dimensional vector so that all the details about the report are stored clearly and projected into n-dimensional space, which is geometrically represented as a dense shape and is taken care of by chi-square PCA model, to reduce the low latent space vectors. The covariance, a relation between the dimensions and observations, helps the PCA reduce the number of features considered for constructing a classifier. The content in the images is categorical data, so the computation of statistical measures is done by chi-square test. The model has to predict one output out of 4 class labels, so; the last constructs, with the help of a densely connected layer, attach with softmax activation function, which is popular in handling the multi-classification, reduces the burden of the overfitting problem.

In [14], Stephane Schwarz et al. designed EMET, which considers users' responses for a particular post by performing multi-classification as true, false, and unknown. These three values are projected onto a latent space using NLP techniques; corresponding transformers did generate. The author has chosen LS representation to draw the inferences, decisions, and finally to construct a two-dimensional matrix between the post, comments, and users. The input signal transformation has marked its place in the NLP world because it generates bitextual pairs, draws the semantic relations between the closest words, and answers for a particular search query 
displays with the rules that match with expert system shells. The model has used a hyper tuned CNN to classify the images by finding the best number of filters, strides, filter size, and several neurons.

In [15], SawinderKaur et al. used the concept of voting at multi-levels to identify fake news. This model has experimented with three features extraction techniques implemented as a step in ML algorithms LR and Linear SVC. All the traditional cleaning steps take care of initial processing, and mean values for different types of articles present in different datasets are measured. The System generates features; a sparse matrix does publish, using different computations like TF-IDF, hashing, and count vectors. In the TF-IDF vector, the relationship does base on the count and its associated weight; for every word, the probability of word occurrence in each document is represented in the interaction cell.

In contrast, the count vector is a numerical representation. The hashing is a novel approach implemented to store the values in buckets based on the calculated remainder value; the major advantage of this approach is that it efficiently handles the memory space during the extraction process. The reason for selecting a multi-level algorithm is to reduce the training time and to generate the outputs in parallel by using the voting classifier. The voting classifier picks the top 3 algorithms with high false rates and ensemble them because the higher the false rate, they are weak the classifiers. The model once again constructs the soft voting classifier and predicts the label based on their false positive rate from the obtained results.

In [16], Mohammad HadiGoldani et al. incrementally designed a margin loss CNN. This research aims to create a less error-prone function that reduces the cross-entropy generated by the softmax activation function. In general, the softmax functions don't adapt to the new environment, so to overcome this drawback; this research has extended the activation function with reinforcement learning. This mechanism helps in generating related featured during the process of adaptive learning. The model uses intracluster property while performing the compaction technique and inters cluster property for separating the dissimilar characteristics. The defined lambda value specifies the margin value empirically predicts the class labels.

\section{A. Gaps Identified}

Few researchers worked on the textual data to identify the fake news and few worked on both images and text. The working on images and text involves complex operations so to simplify the model, it is needed a design an architecture which reduces the cost of operations that are involved in image processing. It is observed that most of the systems find difficulty in finding the semantic relation between the different images along with their text. The popular technique for feature reduction is PCA, but it cannot handle the images where the text is embedded in the image so the usage of variant auto encoders and decoders might help the system to expose both the image features and textual features. Most of the researchers utilized the predefined architectures due to which sometimes it may suffer from bottle neck problem. The solution to this problem might be provided with the usage of transfer learning.

\section{RESULTS AND DISCUSSION}

All the existing systems have successfully implemented the detection of fake news in different social media platforms. Table II compares the methods used in previous case studies and their usage of datasets and identifies the limitations found to address the research gap for implementing a better system with efficient metrics in terms of all objective functions that a deep learning system needs.

To understand the flaws and advantages in terms of various metrics, article has tabulated all the results obtained from different old research systems in Table III.

TABLE II. COMPARATIVE STUdY ON EXISTING SYSTEM OF FAKE NEWS DETECTORS

\begin{tabular}{|c|c|c|c|c|}
\hline Author & $\begin{array}{l}\text { Approach Implemented } \\
\text { for Feature Extraction }\end{array}$ & $\begin{array}{l}\text { Algorithms Implemented for } \\
\text { Classification }\end{array}$ & Dataset (s) Used & Limitations \\
\hline Zhou[1] & Multi-Modal & $\begin{array}{l}\text { Similarity Aware Capture } \\
\text { Model }\end{array}$ & $\begin{array}{l}\text { 1. PolitiFact } \\
\text { 2. GossipCop }\end{array}$ & $\begin{array}{l}\text { Articles that are in pair format from different } \\
\text { sources but published differently are not taken } \\
\text { care }\end{array}$ \\
\hline Shah[2] & Cultural Algorithm & SVM & $\begin{array}{l}\text { 1. Weibo } \\
\text { 2. Twitter }\end{array}$ & $\begin{array}{l}\text { The model cannot handle the complex relations } \\
\text { between the text and visual, if any }\end{array}$ \\
\hline Giachanou[3] & $\begin{array}{l}\text { Google Word Embedding } \\
\text { Layer + LBP }\end{array}$ & Inception+Xception & $\begin{array}{l}\text { 1. PolitiFact } \\
\text { 2. GossipCop } \\
\text { 3.MediaEval }\end{array}$ & $\begin{array}{l}\text { It is not able to produce the decision with neutral } \\
\text { polarity. }\end{array}$ \\
\hline Mangal[4] & $\begin{array}{l}\text { Textual- word2vec } \\
\text { Visual- VGG Net }\end{array}$ & LSTM+ Cosine Similarity & Twitter & Semantically irrelevant data didn't consider here. \\
\hline Zhang[5] & Multi-Modal & Domain Classifier (BDANN) & $\begin{array}{l}\text { 1. Weibo } \\
\text { 2. Weibo Filtered }\end{array}$ & $\begin{array}{l}\text { The domain classification rate further extended } \\
\text { by computing the probability rate }\end{array}$ \\
\hline Sahoo[6] & Web Crawler in browser & $\begin{array}{l}\text { 1. Group of ML Algorithms } \\
\text { 2. Group of DL Algorithms }\end{array}$ & Own Dataset & $\begin{array}{l}\text { In ML algorithms, ensemble techniques, and the } \\
\text { DL algorithms, Hyper tune parameters can yield } \\
\text { better results. }\end{array}$ \\
\hline Agarwal[7] & $\begin{array}{l}\text { N-gram through } \\
\text { Convolution }\end{array}$ & LSTM & Kaggle & $\begin{array}{l}\text { The dataset with an early annotation mechanism } \\
\text { helps in the good clustering of characteristics. }\end{array}$ \\
\hline Cao J[8] & CNN+word2vec & $\begin{array}{l}\text { LSTM with multiple packages, } \\
\text { AIRD }\end{array}$ & Own Dataset & The Metadata generator takes much more time. \\
\hline $\begin{array}{l}\text { Yin-Fu } \\
\text { Huang[9] }\end{array}$ & $\begin{array}{l}\text { Ensemble LSTM+ N-Gram } \\
\text { CNN }\end{array}$ & Self Adaptive HS & Cross-Domain Dataset & $\begin{array}{l}\text { It deals with only headlines mentioned in the } \\
\text { image. }\end{array}$ \\
\hline
\end{tabular}




\begin{tabular}{|c|c|c|c|c|}
\hline Choudhary[10] & $\begin{array}{l}\text { Syntax+ Semantics+ } \\
\text { Readability+ Correlation }\end{array}$ & $\begin{array}{l}\text { Feature based sequential neural } \\
\text { networks }\end{array}$ & BuzzFeed & $\begin{array}{l}\text { The memory and temporal data should do taken } \\
\text { into consideration for efficient implementation }\end{array}$ \\
\hline Zeng[11] & $\begin{array}{l}\text { Multiple Components } \\
\text { Encoder with Soft } \\
\text { Attention }\end{array}$ & LSTM & $\begin{array}{l}\text { 1.Twitter } \\
\text { 2.Weibo }\end{array}$ & $\begin{array}{l}\text { Usage of GANS can improve the Size and Quality } \\
\text { of the dataset. }\end{array}$ \\
\hline Kaliyar[12] & Tensor Formation & XGBOOST+CNN & $\begin{array}{l}\text { 1.Buzzfeed } \\
\text { 2.PolitiFact }\end{array}$ & $\begin{array}{l}\text { The activation function selected suffers from a } \\
\text { zero gradient problem. }\end{array}$ \\
\hline M. Umer[13] & PCA & CNN+LSTM & FNC & $\begin{array}{l}\text { PCA is the traditional approach. Advanced } \\
\text { techniques like variational autoencoders can } \\
\text { extract high-quality and related features. }\end{array}$ \\
\hline S. Schwarz[14] & Signal transformations & EMET & Twitter & $\begin{array}{l}\text { Data augmentation techniques did fail in the case } \\
\text { of an image with side annotations. }\end{array}$ \\
\hline Kaur[15] & $\begin{array}{l}\text { TF-IDF, Count, and } \\
\text { hashing vectors }\end{array}$ & Multi-level voting system & $\begin{array}{l}\text { 1. NewsTrends } \\
\text { 2.Kaggle } \\
\text { 3. Reuters }\end{array}$ & $\begin{array}{l}\text { Further research should control Impulsive data at } \\
\text { an early stage. }\end{array}$ \\
\hline Goldani[16] & Embedding Layer & CNN & LIAR & The embedded layer used is static. \\
\hline Kaliyar[17] & $\begin{array}{l}\text { Glove+Pre-trained word } \\
\text { embeddings }\end{array}$ & Deeper CNN: HCNN+HLSTM & Kaggle & $\begin{array}{l}\text { Instead of traditional neural networks, residual or } \\
\text { transfer learning neural networks can handle } \\
\text { complex relations and associations. }\end{array}$ \\
\hline Hamdi T[18] & Twitter API & Node2vec selection classifier & CREDBANK & $\begin{array}{l}\text { It has to generate synthetic works to handle the } \\
\text { abnormal relations findings. }\end{array}$ \\
\hline Masciari E[19] & Word Embedding & Google BERT & $\begin{array}{l}\text { 1. LIAR } \\
\text { 2.PolitiFact }\end{array}$ & $\begin{array}{l}\text { Diffusion mechanisms integrated with BERT } \\
\text { improve the space complexity of the environment. }\end{array}$ \\
\hline A. Agarwal[20] & N-Grams & $\begin{array}{l}\text { Different Algorithms in ML \& } \\
\text { DL }\end{array}$ & LIAR+Kaggle & $\begin{array}{l}\text { Hyper turned LSTM with enhanced activation } \\
\text { functions is needed. }\end{array}$ \\
\hline
\end{tabular}

TABLE III. METRICS COMPARISON TABLE

\begin{tabular}{|c|c|c|c|c|c|}
\hline Authors & Dataset & Accuracy & Precision & Recall & F1-Score \\
\hline \multirow{2}{*}{ [1] } & PolitiFact & 0.874 & 0.889 & 0.903 & 0.896 \\
\hline & GossipCop & 0.838 & 0.857 & 0.937 & 0.895 \\
\hline \multirow{2}{*}{ [2] } & Weibo & 0.891 & 0.873 & 0.822 & 0.932 \\
\hline & Twitter & 0.798 & 0.791 & 0.833 & 0.760 \\
\hline \multirow{3}{*}{ [3] } & PolitiFact & 0.925 & 0.911 & 0.911 & 0.911 \\
\hline & GossipCop & 0.829 & 0.815 & 0.815 & 0.815 \\
\hline & MediaVal & 0.622 & 0.885 & 0.885 & 0.885 \\
\hline [4] & Twitter & 0.91 & 0.909 & 0.913 & 0.910 \\
\hline \multirow{2}{*}{ [5] } & Weibo & 0.85 & 0.869 & 0.836 & 0.852 \\
\hline & Weibo Filtered & 0.865 & 0.850 & 0.920 & 0.88 \\
\hline [7] & Kaggle & 0.91 & 0.97 & 0.925 & 0.946 \\
\hline [9] & Cross Domain & 0.916 & 0.964 & 0.918 & 0.929 \\
\hline [10] & BuzzFeed & 0.841 & 0.77 & 0.84 & 0.812 \\
\hline \multirow{2}{*}{ [11] } & Twitter & 0.772 & 0.813 & 0.741 & 0.775 \\
\hline & Weibo & 0.839 & 0.867 & 0.784 & 0.828 \\
\hline \multirow{2}{*}{ [12] } & BuzzFeed & 0.856 & 0.833 & 0.869 & 0.851 \\
\hline & PolitiFact & 0.886 & 0.821 & 0.846 & 0.84 \\
\hline [13] & FNC & 0.978 & 0.974 & 0.982 & 0.978 \\
\hline [14] & Twitter & 0.940 & 0.913 & 0.912 & 0.916 \\
\hline \multirow{3}{*}{ [15] } & NewsTrends & 0.93 & 0.958 & 0.916 & 0.937 \\
\hline & Kaggle & 0.98 & 0.988 & 0.98 & 0.983 \\
\hline & Reuters & 0.961 & 0.968 & 0.95 & 0.958 \\
\hline [16] & LIAR & 0.99 & 0.967 & 0.945 & 0.95 \\
\hline [17] & Kaggle & 0.983 & 0.994 & 0.96 & 0.98 \\
\hline [18] & CredBank & 0.98 & 0.98 & 0.98 & 0.98 \\
\hline \multirow{2}{*}{ [19] } & LIAR & 0.588 & 0.565 & 0.449 & 0.528 \\
\hline & PolitiFact & 0.448 & 0.443 & 0.495 & 0.473 \\
\hline [20] & LIAR+Kaggle & 0.97 & 0.965 & 0.951 & 0.958 \\
\hline
\end{tabular}




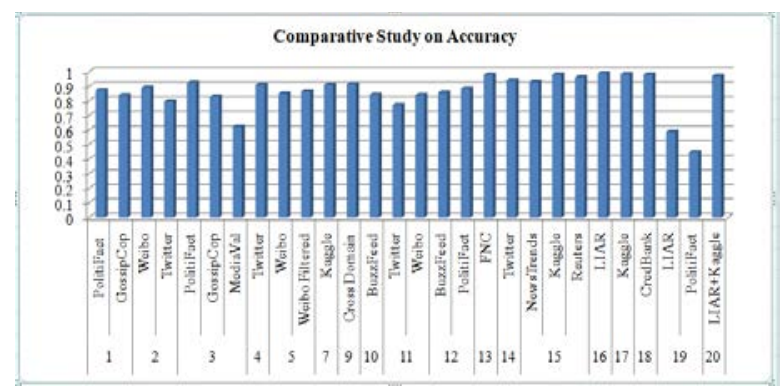

Fig. 5. Comparative Study on Accuracy Metric.

Fig. 5 describes the comparative study on accuracy metric, and from the figure, it has been found that author [16] has achieved nearly $99.9 \%$ accuracy on the Liar dataset, which is highest in terms of both similar and non-similar datasets [20][21]. The author has implemented a simple static word embedding technique to extract the features. Still, in further research, one can try to implement the non-static and redundant dimensions to improve the learning rate and other adaptability parameters because accuracy alone cannot justify the model's reliability.

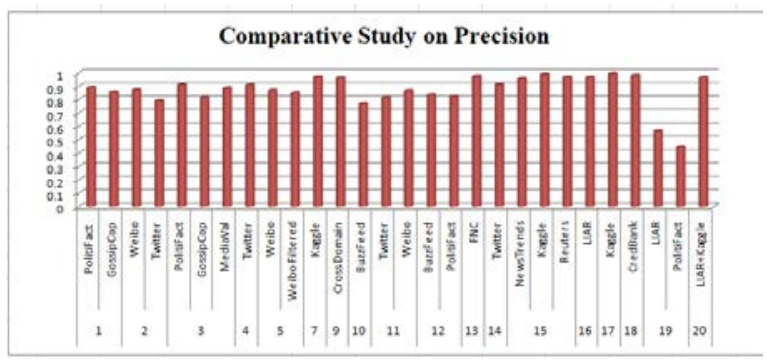

Fig. 6. Comparative Study on Precision Metric.

Fig. 6 clearly states that the author [17] [22-24] has achieved the highest precision rate, "99.4\%," by working on the Kaggle dataset. The model has successfully implemented hyper-turning of estimators inclemently, which involves checking of $2^{* *} \mathrm{n}$ combinations, increasing the execution and training time. So, in further research, the selection of combinations can be reduced by creating a transfer learning environment.

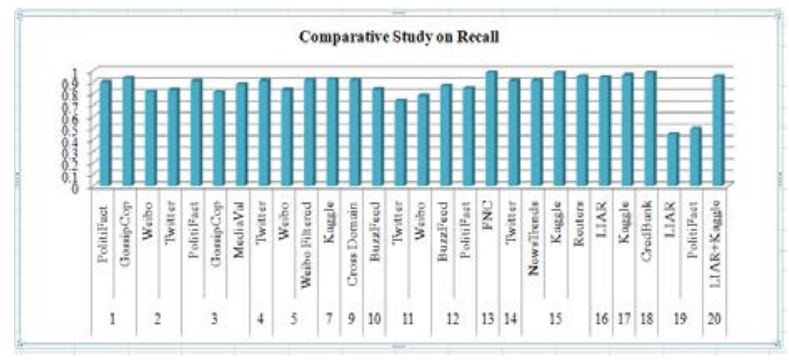

Fig. 7. Comparative Study on Recall Metric.

Fig. 7 states that the author [13] has achieved the highest recall rate, "98.2\%", by working on the FNC dataset. Still, it has applied traditional PCA algorithms for feature reduction and extraction, which further improves by defining the ensemble or variational autoencoders, which has proved its capability in extracting the visual features from multimedia content.

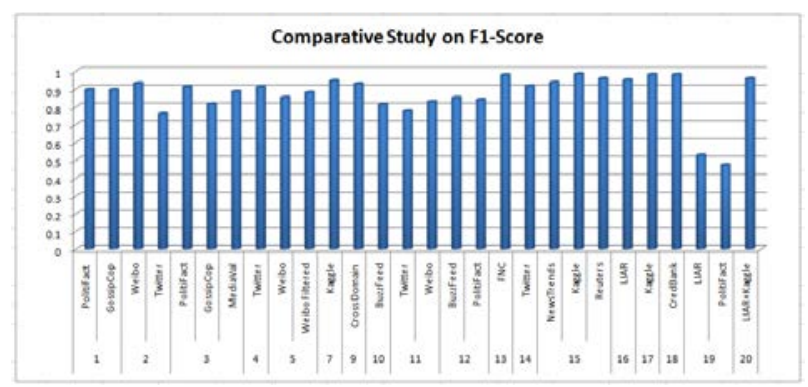

Fig. 8. Comparative Study on F1-Score Metric.

Fig. 8 showed that the author [15] had obtained an F1score of "98.3\%" on the Kaggle dataset by experimenting with three types of feature vectors and designed its classification algorithm, but it suffered when the data, either images or text are in impulsive nature so, in the early stages of model designing, one has to take care of these type of persistent relations.

\section{OBSERVATIONS}

Table II inferences that [19], [3c], and [11] are inferior in performance in terms of all metrics. So this problem can be addressed by neural style transfer learning GANS because they can handle both images and text very effectively. The reason for selecting GANS does explain in two situations, one where the pre-processing step fails to separate the visual and text and the second where the number of images is significantly less to design a model. Next, the proposed system from the above study identified that [16] and [17] are the top two systems in identifying phony news. From these limitations, the system identified the second research gap has to take care of feature extraction with the latest deep learning techniques like autoencoders, by hyper turning and by integrating an enhanced activation function at its output layer, which is dense as fully connected layers. So that the high complex features propagate to the next layers as a simple linear input, finally, to improve the overall capability of the system, the latest transfer learning approach implements by incorporating residual neural networks with pre-trained models to reduce the time for training and to define the correct lambda values to predict the correct output labels associated with the annotated images.

\section{CONCLUSION}

This extensive literature survey helps the system to identify the drawbacks associated with the previous methodologies. From this study, the system has identified that Natural Language Processing tools like N-grams, SVD decomposition, Lemmatization's and other simple processing techniques are efficient to work on the text extracted from the images. To work with the images, instead of simple CNN techniques, it is better to design modified auto encoders which can efficiently solve the problem of bottle neck in linear amount of time. The feature extraction is the crucial step in fake news detection because the text objects in the image play a vital role in deciding whether it is real or fake news. For the classification of news, it is better to implement ensemble algorithms or Meta classifiers, which achieves good accuracy and true positive rates. The ensemble algorithms have proved 
their efficiency in terms of AUC and Kappa Statistics measurement. In future, to detect fake news detection, the proposed research first implements GANs to increment the size of data then it extracts the features using AEDECNN and then it performs classification using ensemble mechanism.

\section{GLOSSARY}

\begin{tabular}{|l|l|l|}
\hline S.No & Term & Full Form \\
\hline 1 & CNN & Convolution Neural Networks \\
\hline 2 & GAN & Generative Adversial Networks \\
\hline 3 & LSTM & Long Short Term Memory \\
\hline 4 & RBFNN & Radial Bias Function Neural Network \\
\hline 5 & MLP & Multi Layer Perceptron \\
\hline 6 & SVD & Single Value Decomposition \\
\hline 7 & AUC & Area Under Characteristics \\
\hline
\end{tabular}

REFERENCES

[1] Zhou, Xinyi, et al. "SAFE: Similarity-Aware Multi-Modal Fake News Detection.” Advances in Knowledge Discovery and Data Mining, edited by Hady W. Lauw et al., vol. 12085, Springer International Publishing, 2020, pp. 354-67. DOI.org (Crossref), doi:10.1007/978-3-030-474362_27.

[2] Shah, P., \&Kobti, Z. (2020). Multimodal fake news detection using a Cultural Algorithm with situational and normative knowledge. 2020 IEEE Congress on Evolutionary Computation (CEC). doi:10.1109/cec48606.2020.9185643.

[3] Giachanou A., Zhang G., Rosso P. (2020) Multimodal Fake News Detection with Textual, Visual and Semantic Information. In: Sojka P., Kopeček I., Pala K., Horák A. (eds) Text, Speech, and Dialogue. TSD 2020. Lecture Notes in Computer Science, vol 12284. Springer, Cham. https://doi.org/10.1007/978-3-030-58323-1_3.

[4] Mangal, D., \& Sharma, D. K. (2020). Fake News Detection with Integration of Embedded Text Cues and Image Features. 2020 8th International Conference on Reliability, Infocom Technologies and Optimization (Trends and Future Directions) (ICRITO). doi:10.1109/icrito48877.2020.9197817.

[5] Zhang, T., Wang, D., Chen, H., Zeng, Z., Guo, W., Miao, C., \& Cui, L. (2020). BDANN: BERT-Based Domain Adaptation Neural Network for Multi-Modal Fake News Detection. 2020 International Joint Conference on Neural Networks (IJCNN). doi:10.1109/ijcnn48605.2020.9206973.

[6] Sahoo, S. R., \& Gupta, B. B. (2021). Multiple features based approach for automatic fake news detection on social networks using deep learning. Applied Soft Computing, 100, 106983. https://doi.org/10.1016/j.asoc.2020.106983.

[7] Agarwal, A., Mittal, M., Pathak, A. et al. Fake News Detection Using a Blend of Neural Networks: An Application of Deep Learning. SN COMPUT. SCI. 1, 143 (2020). https://doi.org/10.1007/s42979-02000165-4.

[8] Cao J., Qi P., Sheng Q., Yang T., Guo J., Li J. (2020) Exploring the Role of Visual Content in Fake News Detection. In: Shu K., Wang S., Lee D., Liu H. (eds) Disinformation, Misinformation, and Fake News in Social Media. Lecture Notes in Social Networks. Springer, Cham. https://doi.org/10.1007/978-3-030-42699-6_8.

[9] Yin-Fu Huang, Po-Hong Chen, Fake news detection using an ensemble learning model based on Self-Adaptive Harmony Search algorithms, Expert Systems with Applications, Volume 159,2020, 113584, ISSN 0957-4174,https://doi.org/10.1016/j.eswa.2020.113584.

[10] Choudhary, A., \& Arora, A. (2021). Linguistic feature based learning model for fake news detection and classification. Expert Systems with Applications, 169, 114171. https://doi.org/10.1016/j.eswa.2020.114171'.
[11] Zeng, J., Zhang, Y., \& Ma, X. (2021). Fake news detection for epidemic emergencies via deep correlations between text and images. Sustainable Cities and Society, 66, 102652. https://doi.org/10.1016/j.scs.2020. 102652.

[12] Kaliyar, R.K., Goswami, A. \& Narang, P. DeepFakE: improving fake news detection using tensor decomposition-based deep neural network. J Supercomput 77, 1015-1037 (2021). https://doi.org/10.1007/s11227020-03294-y.

[13] M. Umer, Z. Imtiaz, S. Ullah, A. Mehmood, G. S. Choi and B. -W. On, "Fake News Stance Detection Using Deep Learning Architecture (CNNLSTM)," in IEEE Access, vol. 8, pp. 156695-156706, 2020, doi: 10.1109/ACCESS.2020.3019735.

[14] S. Schwarz, A. Theóphilo and A. Rocha, "EMET: Embeddings from Multilingual-Encoder Transformer for Fake News Detection," ICASSP 2020 - 2020 IEEE International Conference on Acoustics, Speech and Signal Processing (ICASSP), 2020, pp. 2777-2781, doi: 10.1109/ICASSP40776.2020.9054673.

[15] Kaur, S., Kumar, P. \&Kumaraguru, P. Automating fake news detection system using multi-level voting model. Soft Comput 24, 9049-9069 (2020). https://doi.org/10.1007/s00500-019-04436-y.

[16] Goldani, M. H., Safabakhsh, R., \&Momtazi, S. (2021). Convolutional neural network with margin loss for fake news detection. Information $\begin{array}{llll}\text { Processing \& } \quad \text { Management, } & \text { 58(1), }\end{array}$ https://doi.org/10.1016/j.ipm.2020.102418.

[17] Kaliyar, R. K., Goswami, A., Narang, P., \& Sinha, S. (2020). FNDNet A deep convolutional neural network for fake news detection. Cognitive Systems Research, 61, 32-44. https://doi.org/10.1016/j.cogsys.2019.12.005.

[18] Hamdi T., Slimi H., Bounhas I., Slimani Y. (2020) A Hybrid Approach for Fake News Detection in Twitter Based on User Features and Graph Embedding. In: Hung D., D’Souza M. (eds) Distributed Computing and Internet Technology. ICDCIT 2020. Lecture Notes in Computer Science, vol 11969. Springer, Cham. https://doi.org/10.1007/978-3-03036987-3_17.

[19] Masciari E., Moscato V., Picariello A., Sperli G. (2020) A Deep Learning Approach to Fake News Detection. In: Helic D., Leitner G., Stettinger M., Felfernig A., Raś Z.W. (eds) Foundations of Intelligent Systems. ISMIS 2020. Lecture Notes in Computer Science, vol 12117. Springer, Cham. https://doi.org/10.1007/978-3-030-59491-6_11.

[20] laiahKavati, A. Mallikarjuna Reddy, E. Suresh Babu, K. Sudheer Reddy, RamalingaSwamyCheruku,Design of a fingerprint template protection scheme using elliptical structures,ICT Express,Volume 7, Issue 4,2021,Pages 497-500,ISSN 24059595,https://doi.org/10.1016/j.icte.2021.04.001.

[21] Ayaluri MR, K. SR, Konda SR, Chidirala SR. 2021. Efficient steganalysis using convolutional auto encoder network to ensure original image quality. PeerJ Computer Science 7:e356 https://doi.org/10.7717/peerj-cs.356.

[22] A. M. Reddy, V. V. Krishna, L. Sumalatha and S. K. Niranjan, "Facial recognition based on straight angle fuzzy texture unit matrix," 2017 International Conference on Big Data Analytics and Computational Intelligence (ICBDAC), Chirala, 2017, pp. 366-372, doi: 10.1109/ICBDACI.2017.8070865. (C 5).

[23] M. Reddy, K. SubbaReddy and V. V. Krishna, "Classification of child and adulthood using GLCM based on diagonal LBP," 2015 International Conference on Applied and Theoretical Computing and Communication Technology (iCATccT), Davangere, 2015, pp. 857-861, doi: 10.1109/ICATCCT.2015.7457003.

[24] R. T, G. Sirisha and A. M. Reddy, "Smart Healthcare Analysis and Therapy for Voice Disorder using Cloud and Edge Computing," 2018 4th International Conference on Applied and Theoretical Computing and Communication Technology (iCATccT), Mangalore, India, 2018, pp. 103-106, doi: 10.1109/iCATccT44854.2018.9001280. 